\title{
Femtosecond laser inscribed phase masks for fibre Bragg grating sensor inscription
}

\author{
Graham C. B. Lee ${ }^{a}$, Kyriacos Kalli ${ }^{+b}$, Charalambos Koutsides ${ }^{b}$, Zhijun Yan ${ }^{\text {a }}$, Dotun Adebayo ${ }^{\text {a }}$, \\ Michael Komodromos ${ }^{c}$, Kate Sugden $*^{\mathrm{a}}$ \\ ${ }^{a}$ Aston Institute of Photonic Technologies, Aston University, Birmingham, UK; \\ ${ }^{\mathrm{b}}$ Nanophotonics Research Laboratory, Cyprus University of Technology, 3036, Lemessos, Cyprus; \\ ${ }^{c}$ Frederick University, Filokyprou, 7 Nicosia 1036, Cyprus
}

\begin{abstract}
We present femtosecond laser inscribed phase masks for the inscription of Bragg gratings in optical fibres. The principal advantage is the flexibility afforded by the femtosecond laser inscription, where sub-surface structures define the phase mask period and mask properties. The masks are used to produce fibre Bragg gratings having different orders according to the phase mask period. The work demonstrates the incredible flexibility of femtosecond lasers for the rapid prototyping of complex and reproducible mask structures. We also consider three-beam interference effects, a consequence of the zeroth-order component present in addition to higher-order diffraction components.
\end{abstract}

Keywords: Phase masks, femtosecond laser, inscription, laser-material interaction, fibre Bragg gratings, Talbot effect

\section{INTRODUCTION}

The use of phase masks for the production of fibre gratings was spurred by research such as that of Bennion $e t$ al who first demonstrated side-etched gratings in 1986 [1]. Later work by Hill et al [2] provided the necessary impetus for the mass production of fibre Bragg gratings (FBGs). The role of the phase mask is to spatially modulate the UV-writing beam to produce a suitable interference pattern in the fibre core that defines the extent and strength of the Bragg grating. Phase masks are typically surface relief structures, with the gratings most often etched in low loss, fused silica via an electron-beam or holographic process. A key advantage of electron-beam lithography is the writing of complicated patterns, potentially offering quadratic chirps and Moire patterns written directly into the mask's structure. However, lithographically produced phase masks are generated by typically stitching together small subsections $(400 \mu \mathrm{m} \times 400 \mu \mathrm{m})$ of periodic corrugations on the mask substrate to fabricate larger phase structures. An error in the precise positioning of the various subsections results in what is commonly referred to as stitching error [3]. Holographically produced phase masks have no stitch error. The masks act as precision diffraction gratings that upon irradiation by a monochromatic source divide the light evenly between two or more orders. These created orders interfere in the area behind the phase mask where they overlap and this interference pattern is recorded in the fibre to create the FBG. An area that is particularly topical in recent years is the use of femtosecond ( $\mathrm{fs}$ ) lasers to induce refractive index changes or ablation in transparent materials. A focused femtosecond laser pulse can fundamentally change a material's physical properties through strong non-linear absorption of the laser energy, allowing for the fabrication of intricate microstructures on the surface of opaque materials, or within the bulk volume of optically transparent glass or polymeric materials. In this work we present the improvement of phase masks written with the point by point femtosecond inscription $[4,5]$. The masks are used to fabricate Bragg gratings in fibres. The fabrication method offers the potential for rapid modification of the mask properties by either manipulating the coding of the sample motion relative to the inscription laser beam and/or by accurately controlling the laser parameters, such as the pulse duration, repetition rate and the laser pulse energy. Our method allows for large area masks without the requirement to stitch together small subsections.

+ kkalli@cytanet.com.cy, kyriacos.kalli@cut.ac.cy; phone +35725002609; www.cut.ac.cy

* k.sugden@aston.ac.uk; phone +441212043498; www.aston.ac.uk 


\subsection{Phase mask inscription}

A series of diffraction patterns were femtosecond-laser inscribed in the bulk volume of pure fused-silica substrates, approximately $50 \mu \mathrm{m}$ below the surface. The uniformity of the substrate was found to be critical in the reproducibility of the masks. To ensure that this was the case we chose a glass known to be highly transmissive from a wavelength range of $193 \mathrm{~nm}$ to $693 \mathrm{~nm}$, thereby making the masks suitable for the production of FBGs. The fs laser writing setup consisted of an Amplitude Systemes s-Pulse HP, producing sub $500 \mathrm{fs}$ laser pulses at a centre wavelength of $1026 \mathrm{~nm}$, and repetition rate of $100 \mathrm{kHz}$. The laser energy ranged from $1 \mu \mathrm{J}$ to $8 \mu \mathrm{J}$ at the laser exit. All inscriptions were conducted using a $100 \mathrm{X}$ objective (Mititoyo MPlan Apo NIR Series) with a numerical aperture of 0.5. This was chosen as it provides a long working distance with a high NA thus generating a small and highly accurate focal spot that the structures written required. The accurate nature of the spot through the sample due to the large NA and working distance are critical to the direct write nature of the work. The FWHM of the laser spot size incident on the samples was approximately $1.5 \mu \mathrm{m}$. The samples were mounted on an Aerotech motion control system (x-y ABL 1000 air bearing stages) that was controlled with custom coding. The repeatability and precision of these stages allows this work to be carried out with minimal stitch errors and with the nanometre accuracy required to obtain the precise lines written in the fused silica substrates. The samples were secured perpendicular to the incident femtosecond laser beam. Low stress mounting of the samples ensured unwanted effects that could change the uniformity of the written structure.

The best results were achieved for a laser energy at the sample of $350 \mathrm{~nJ}$ per pulse, at a translation speed of $2 \mathrm{~mm} / \mathrm{s}$. This proved to be a good compromise to produce reliable and clear lines for a reasonable mask inscription time. Fig. 1 (a) offers a close up of the inscription process, with the laser plasma within the bulk volume of the substrate clearly visible. In Fig. 1(b) we observe the series of masks that were inscribed, whereas Fig. 1 (c) shows a transmission microscope image of a typical mask line structure for a period of approximately $2 \mu \mathrm{m}$. The actual masks periods were chosen to provide FBG spectra in the $1550 \mathrm{~nm}$ transmission window. Hence we selected periods of $1071.18 \mathrm{~nm}$ for the mask designated M10, and $2142.36 \mathrm{~nm}$ and $3180.00 \mathrm{~nm}$ for the masks designated M8 and M12, respectively. The period affected the inscription time, varying from one and a half hours to five hours, accordingly.

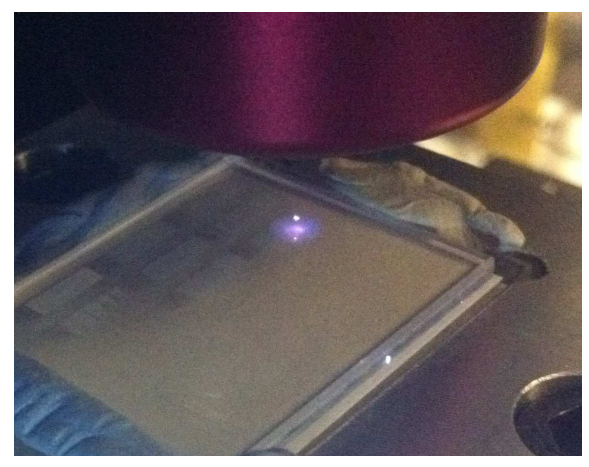

(a)

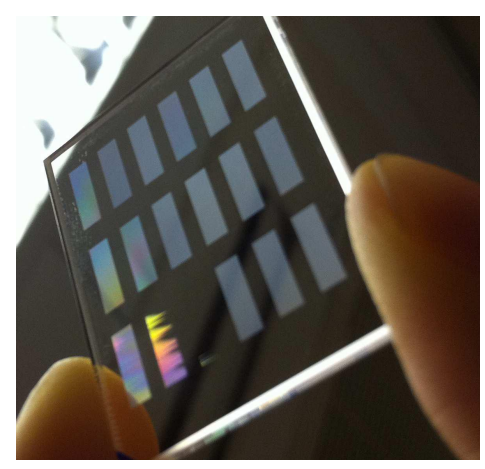

(b)

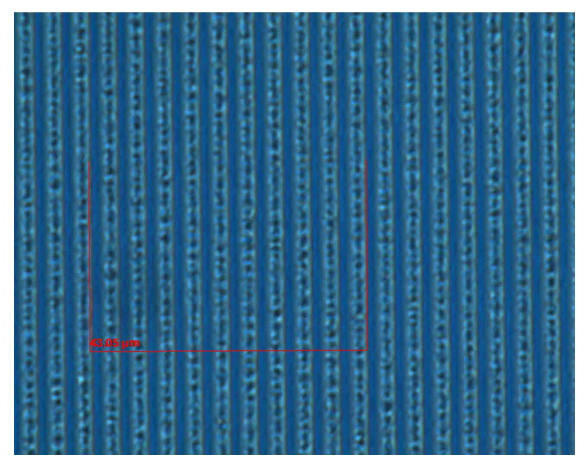

(c)

Fig. 1 (a) close up of the inscription process showing the laser plasma within the substrate, (b) a series of inscribed masks, (c) transmission microscope image of a typical mask line structure for a period of approximately $2 \mu \mathrm{m}$.

The samples were viewed with an optical microscope (Fig. 1 (c)) and an optical profilometer (WYKO 9100NT), Fig. 2. As we are viewing subsurface structures, our interest in using the optical profilometer is to measure the period and view the consistency of the line structure amplitude; hence we use an intensity mode, where absolute values are not measured with regard to amplitude. Fig. 2 (a) shows a typical 3-D plot of the mask, whereas Fig. 2 (b) shows a line section across the mask, from which we observe that the mask has both excellent consistency in amplitude and period.

In Fig. 3 we observe the potential for the fs inscription method, where we observe the actual diffraction performance of the mask, from which we can discern that it is behaving as a phase mask, when the mask is illuminated by a $244 \mathrm{~nm}$ UV laser, as typically used for FBG inscription. 

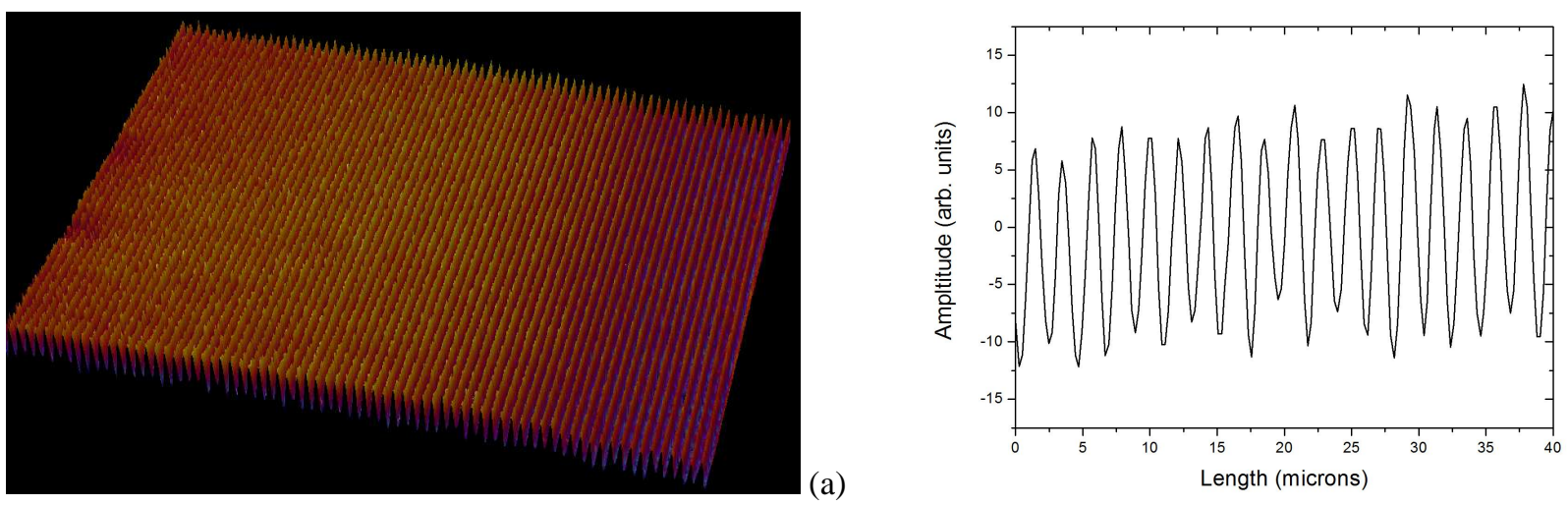

Fig. 2. Typical optical profilometer 3-D plot of the mask, (b) a line section of the mask; we observe that the mask has both excellent consistency in amplitude and period.

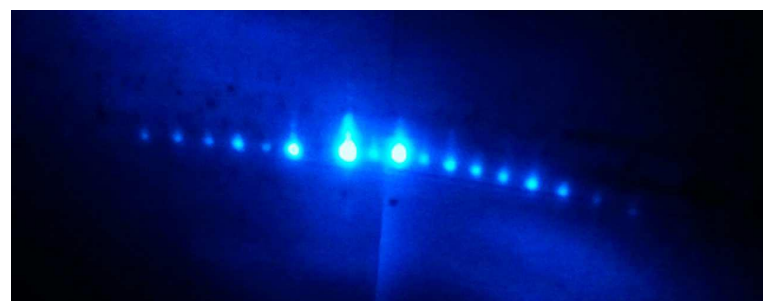

Fig. 3. The diffraction performance of a typical femtosecond-laser inscribed mask, when illuminated by a UV laser, as typically used for FBG inscription.

\subsection{FBG inscription}

A series of FBGs were inscribed in hydrogenated Fibrecore PS-1250/1500, a B/Ge co-doped optical fibre. The fibres were hydrogenated at $200 \mathrm{bar}$ and $80^{\circ} \mathrm{C}$ for 48 hours and stored for a short period at $-40^{\circ} \mathrm{C}$ before FBG inscription. Table 1 summarises the FBGs that were inscribed using the phase masks. The grating transmission spectra were recorded using a broadband light source and optical spectrum analyser. Fig. 4 shows a transmission spectrum for a FBG UVwritten using mask M12 and having a 7- $d B$ notch depth.

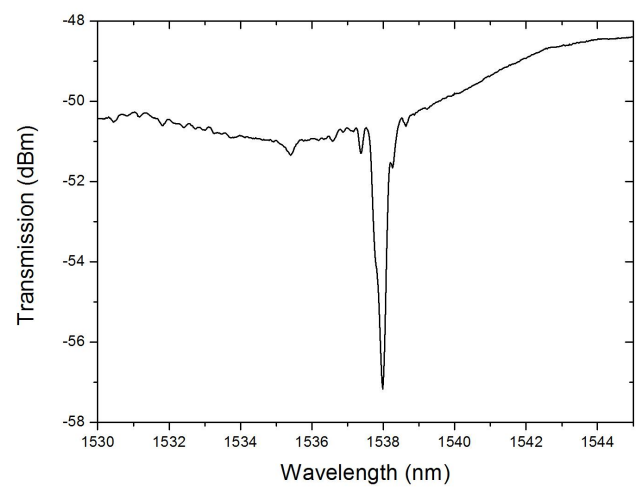

Fig. 4. FBG inscribed using the femtosecond inscribed phase mask.

Table 1. FBG inscription parameters

\begin{tabular}{|c|c|c|c|c|c|}
\hline Grating & Mask ID & Mask Type & UV Laser Power $(W)$ & Scans & Speed $(\mathrm{mm} / \mathrm{s})$ \\
\hline FBG4 & M12 & $3^{\text {rd }}$ order $1550 \mathrm{~nm}$ & 0.1 & 1 & 0.05 \\
\hline FBG5 & M10 & $1^{\text {st }}$ order $1550 \mathrm{~nm}$ & 0.1 & 3 & 0.05 \\
\hline FBG7 & M8 & $2^{\text {nd }}$ order $1550 \mathrm{~nm}$ & 0.1 & 1 & 0.05 \\
\hline
\end{tabular}




\subsection{Zeroth order suppression}

With regard to the inscription of the Bragg gratings using the femtosecond inscribed masks, one needs to consider the effect that the zeroth-order has on any interference pattern that is generated by the mask. This is essentially three-beam interference that results between the non-zero suppression of the zeroth-order with the different diffracted order pairs. Higher order contributions are typically ignored as they can be minimized by increasing the spacing between the fiber and the mask. Hence when using the femtosecond inscribed masks for Bragg grating inscription we will tend to inscribe second order Bragg gratings instead of a conventional first order Bragg grating. This is not in itself a major drawback, but pertains from the fact that we have yet to optimise the effective laser induced "etch-depth" of the mask and its relation to the UV wavelength used for the Bragg grating inscription. This is under development and we anticipate far greater diffraction efficiencies once this issue has been resolved.

\section{CONCLUSION}

We have presented improved femtosecond-laser inscribed sub-surface phase/amplitude masks for the production of FBGs in hydrogenated optical fibre. The work demonstrates the proof of concept and flexibility for the use of femtosecond lasers to make complex and reproducible masks. This approach to fabricating masks enables the patterns to be below the surface which is helpful in the protection of the tooling and reproducibility of gratings due to positioning variations. One does need to account for the effect of the non-zero contribution of the strong zeroth-order component that is present in the transmission characteristics of the mask and that modifies the ideal, anticipated interference pattern typically used for Bragg grating inscription. Hence when using the femtosecond inscribed masks for Bragg grating inscription we are inscribing second order Bragg gratings instead of a conventional first order Bragg grating. This is not in itself a major drawback, but the effective laser induced "etch-depth" of the mask needs to be optimised with respect to the UV wavelength used for the Bragg grating inscription. This is under development and we anticipate far greater diffraction efficiencies once this issue has been resolved.

\section{ACKNOWLEDGMENTS}

G. Lee wishes to thank the Engineering and Physical Sciences Research Council (EPSRC) for funding his Ph.D. K. Kalli and M. Komodromos acknowledge the Research Promotion Foundation, Cyprus, for support of this work under the

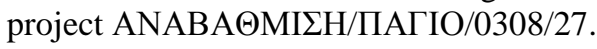

\section{REFERENCES}

[1] I. Bennion, D. C. J Reid, C. J. Rowe, and W. J. Stewart, "High reflectivity monomode-fibre grating filters", Electronics Letters, 22, 341-342 (1986).

[2] K. O. Hill, B. Malo, F. Bilodeau, D. C. Johnson, and J. Albert, "Bragg gratings fabricated in monomode photosensitive optical fibre by UV exposure through a phase mask", Applied Physics Letters, 62 (10), 10351037 (1993).

[3] J. Albert, S. Theriault, F. Bilodeau, D. C. Johnson, K. O. Hill, P. Sixt, and M. J. Rooks "Minimization of phase errors in long fibre Bragg grating phase masks made using electron beam lithography", IEEE Photon. Technol. Lett., 8, 1334-1336 (1996).

[4] G. N. Smith, K. Kalli, I. Bennion, and K. Sugden, "Demonstration of inscription and ablation of phase masks for the production of 1st, 2nd, and 3rd order FBG gratings using a femtosecond laser" in Advanced Fabrication Technologies for Micro/Nano Optics and Photonics II, SPIE Vol. 7205, pp 7205112009.

[5] G. N. Smith, K. Kalli, and K. Sugden "Advances in femtosecond machining and inscription of micro and nano photonic devices" in Frontiers in Guided Wave Optics and Optoelectronics. Bishnu Pal. Ed. InTech, 2010. 